\title{
Effect of Maternal Hypothyroidism on BDNF Expression in Developing Rat Brain
}

\author{
Nemati Hossein ${ }^{1}$, Parivar Kazem ${ }^{1}$, Doroud Delaram ${ }^{2}$, Hayati Roudbari Nasim ${ }^{1} \&$ Nabiuni Mohammad $^{3}$ \\ ${ }^{1}$ Department of Biology, Science and Research Branch, Islamic Azad University, Tehran, Iran \\ ${ }^{2}$ Quality Department, Production and Research Complex, Pasteur Institute of Iran, Tehran, Iran \\ ${ }^{3}$ Department of Animal Biology, Faculty of Biological Sciences, Kharazmi University, Tehran, Iran \\ Correspondence: Kazem Parivar. Department of Biology, Science and Research Branch, Islamic Azad University, \\ Tehran, Iran. E-mail: kazem.parivar13@chmail.ir
}

Received: December 21, 2017

Accepted: January 22, 2018

Online Published: May 5, 2018

doi:10.5539/jmbr.v8n1p31

URL: https://doi.org/10.5539/jmbr.v8n1p31

\begin{abstract}
Thyroid hormone is essential for proper development of brain and modest degrees of developmental thyroid hormone insufficiency can result severe neurological deficits. We used an animal model to study the role of thyroid hormones (THs) during the gestation days and early postnatal days on developing rat brain. Dose-dependent thyroid hormone insufficiency in dams was induced by methimazole (MMI). In the first study-group thyroid hormone deficiency was induced by delivery of methimazole (MMI) to dams via drinking water at the doses of 50,75 and $100 \mathrm{ppm}$ from early gestation (GD 3) until weaning of the pups (PN20). In the second study-group hypothyroid rats were treated with $200 \mu \mathrm{g}$ of thyroxine hormone. Pups were sacrificed on postnatal days (PN) 20 . Maternal blood collected for thyroid hormone analysis. Cerebellum, medulla, hippocampus, cortex, olfactory bulbs were separated from dissected brain and relative expression of $B D N F$ gene transcripts known to play critical roles in developing rat brain, were determined by RT-PCR. Daily body weight in dams and pups, the number of pups at birth, the eye -closure opening day and BDNF expression in brain extract was determined in the preweaning rats as a function of MMI exposure. The results indicate that genes driving important developmental processes during early brain development are sensitive to perturbations of the thyroid axis function.
\end{abstract}

Keywords: Hypothyroidism, Cerebellum, Medulla, Hippocampus, Cortex, Olfactory bulbs, BDNF, Genomics

\section{Introduction}

Thyroid hormones are essential for brain development through specific time windows influencing neurogenesis, neuronal migration, neuronal and glial cell differentiation, myelination, and synaptogenesis (Bernal, 2007). Thyroid hormone action in CNS development is mediated through nuclear receptors (TRs) and integrin $\alpha_{v} \beta_{3}$ (Stenzel et al., 2014) that regulates gene expression. Although many genes in developing rat brain have been reported to be altered by hypothyroidism (Royland et al., 2008) but the impact of severe degrees of perturbation of the thyroid axis function on specific target gene expression known to play critical roles in developing rat brain, has not been detected. The transport of thyroid hormone through the plasma cell membrane is facilitated by the monocarboxylate transporter 8 (MCT8) and MCT10 (SLC16A2 and SLC16A10 genes) (Friesema et al., 2003) the organic anion transporter polypeptides (OATP, especially OATP1C1, SLCO1C1 gene), the large neutral amino acid transporters (LAT-1 and LAT-2, products of the SLC7A5 and SLC7A8 genes), and the sodium/taurocholate co-transporting polypeptide (SLC10A1, or NTCP) (Friesema et al., 2008).

In classic view, the hypothalamic-pituitary-thyroid axis (HPTA) thyroid hormone releasing hormone (TRH) from the hypothalamus stimulates the pituitary to release thyroid stimulating hormone (TSH). TSH acts on thyroid gland receptors to activate synthesis and release of TH (Gilbert \& Lasley, 2013). Given the devastating and irreversible neurological consequence induced during development by thyroid hormone insufficiency, the consequences of subclinical hypothyroidism or hypothyroxinemia would appear to be most acute for developing fetus and neonate (Chen et al., 2014; Morreale de Escobar et al., 2000; Zoeller \& Rovet, 2004). Even modest reductions in thyroid hormones during critical period of brain development produce morphological alterations, synaptic dysfunction, and behavioral impairments (Gilbert \& Zoeller, 2010; Ghassabian et al., 2011; Gilbert et al., 2015). Studies examining the effect of thyroid hormone reduction on gene expression have not produced consistent results. 
Differences in degree, timing and duration of thyroid hormone insufficiency, brain region sampled, age, species of animal model, endpoint assessed (protein or mRNA)( Lasley \& Gilbert, 2011) and the effect of the neonate sexuality on gene expression in developing brain, have complicated the synthesis of finding across studies. Neurotrophins regulate development, maintenance, and function of vertebrate nervous systems. Neurotrophins activate two different classes of receptors, the Trk family of receptor tyrosine kinases and p75NTR, a member of the TNF receptor superfamily. Through these, neurotrophins activate many signaling pathways, including those mediated by ras and members of the cdc- $42 /$ ras/rho G protein families, and the MAP kinase, PI-3 kinase, and Jun kinase cascades. During development, limiting amounts of neurotrophins function as survival factors to ensure a match between the number of surviving neurons and the requirement for appropriate target innervation. They also regulate cell fate decisions, axon growth, dendrite pruning, the patterning of innervation and the expression of proteins crucial for normal neuronal function, such as neurotransmitters and ion channels. These proteins also regulate many aspects of neural function. In the mature nervous system, they control synaptic function and synaptic plasticity, while continuing to modulate neuronal survival (Huang \& Reichardt, 2001). To date limited data are available for thyroid hormone reductions on $B D N F$ gene transcripts in different brain region. This study aimed to evaluate relative expression of thyroid hormone target genes by RT-PCR and have identified that this gene are differentially regulated in different brain region of severely hypothyroid neonate rats in comparison to euthyroid neonate rats.

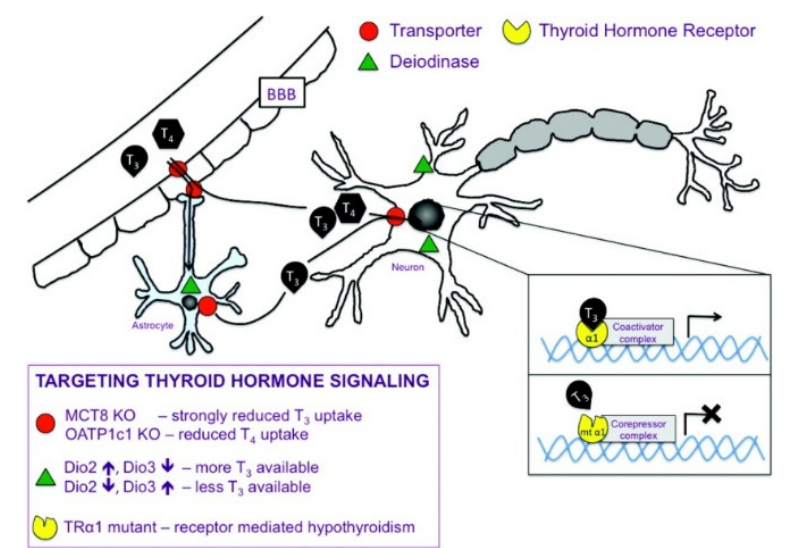

Figure 1. Targeting thyroid hormone signalling in the brain. Several different molecules such as transporters (circle), deiodinases (triangle) and receptors (pacman) are involved in controlling thyroid hormone actions in the brain (Warner \& Mittag, 2012). BBB, blood brain barrier; Dio2/3, deiodinase type II or III; MCT8, monocarboxylate transporter 8; Oatp1c1, organic anion transporting polypeptide 1c1; TR, thyroid hormone receptor

\section{Material and Methods}

\subsection{Hormone Insufficiency during Development}

Pregnant Wistar rats ( $\mathrm{n}=60$ ), weighting 200-260 g, were obtained from Pasteur Institute of Iran on gestation day (GD) 3 and housed individually in standard hanging cages under controlled temperature $\left(24 \pm 1^{\circ} \mathrm{C}\right)$. The housing rooms were maintained on a 12:12 light (06:00-18:00h) - dark(18:00-06:00h) cycle, and standard food and water were available ad libitum. Body weights of dams were monitored throughout gestation and postnatal period; body weights of pups were monitored from PN0 to PN20 and Pup's eye-closure opening day were monitored from PN12 to PN20. Successful delivery day monitored in each treatment group. To induce hypothyroidism, thyroid hormone inhibitor methimazole (Daroupakhsh, Iran) was added to drinking water (50ppm, $75 \mathrm{ppm}, 100 \mathrm{ppm})(\mathrm{n}=12 /$ dose

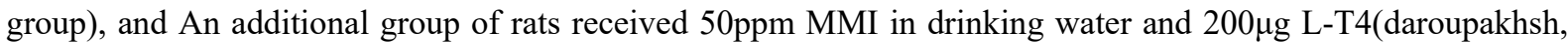
Iran) by gavage ( $\mathrm{n}=12 /$ dose group). Drinking MMI solution was replaced daily with fresh MMI solution. A control group received drinking water without methimazole (MMI). MMI enters the bloodstream, passing through mother's breast milk to the rat pups. All animals treatments began on GD3 and continuing until weaning of the pups (PN20). All animal work was performed according to the IPI Guide for Care and Use of laboratory Animals.

\subsection{Plasma T3, T4 Assays}

Blood was collected directly from the maternal orbital sinus in PN20. Blood samples were centrifuged at $3000 \mathrm{~g}$ for $20 \mathrm{~min}$ at room temperature to obtain the plasma. Plasma was used to analyze levels of thyroid hormones $\mathrm{T}_{3}$, 
$\mathrm{fT}_{4}$, totalT4, and TSH. Serum levels of total $\mathrm{T}_{3}\left(\mathrm{tT}_{3}\right)$ and total $\mathrm{T} 4(\mathrm{tT} 4)$ and free $\mathrm{T}_{4}\left(\mathrm{fT}_{4}\right)$ were measured by ELISA (Padtangostar, Tehran, Iran).

\subsection{BDNF ELISA}

BDNF expression was determined with Rat Brain derived Neurotrophic Factor (BDNF) ELISA kit (Zellbio, Germany) according to the manufacturer's instruction. In each treatment group 5 male neonates, in 100 PPM hypothyroid group 3 male neonates, and 5 female neonates were sacrificed for total mature BDNF analysis. Brains quickly removed and weighed up and added PBS $(\mathrm{pH} 7.4,100 \mathrm{mM})$ and homogenized the sample thoroughly by homogenizer (totally $100 \mathrm{mg}$ tissue $/ 1 \mathrm{ml}$ buffer). The PBS contain anti-protease cocktail (Sigma). Then centrifuged (at 6000 RPM) for approximately 10 minutes. After collecting the supernatant, experiment conducted immediately. Aliquots of each sample lysate were analyzed in duplicate for total protein by The Lowry protein assay and sample mature BDNF protein expression was determined in duplicate in each experimental set of plates.

\subsection{Real-Time Quantitative -PCR}

In each treatment group male and female offspring $(n=3)$ were sacrificed at PN20. Brains were quickly removed and cortex, hippocampus, cerebellum, medulla oblongata and bulbus olfactorius dissected. Total RNA was prepared individually from five brain regions using RNXTM-Plus (Cinnagen, Iran). First strand cDNA was synthesized from $5 \mu \mathrm{g}$ total RNA from one animal using first strand cDNA synthesis (Cinnagen, Iran). Expression levels of the following 10 genes in cDNA samples were quantified by StepOne Real-Time PCR Systems with SYBR Green. Data were analyzed by $2-\Delta \Delta C T$ method using the house keeping gene HPRT as an internal control, as its expression level was not affected by thyroid hormone status, to calculate relative mRNA levels. PCR primers were designed in Oligo7.0 primer analysis software (Molecular Biology Insights) and blasted in https://www.ncbi.nlm.nih.gov. Sequences of the PCR primers are listed in Table 1.

Table 1. Primers for real-time quantitative PCR

\begin{tabular}{lllll}
\hline Gene Symbole & Directions & Sequences of the PCR primers & Function & Genebank ID \\
\hline BDNF & Forward & GATTAGGTGGCTTCATAGGAGAC & Neurotrophic growth factors- Differentiation & NM_001031616.1 \\
& Reverse & AGAACAGAACAGAACAGAACAGG & & S79292.1 \\
HPRT & Forward & CCAGCGTCGTGATTAGTG & housekeeping gene & \\
& Reverse & CGAGCAAGTCTTTCAGTCC & & \\
\hline
\end{tabular}

\subsection{Statistical Analysis}

Statistical analysis was performed using the SPSS statistical package (SPSS Inc., Chicago, IL, USA) for Windows (version 16.0). All data were assessed by one- way ANOVAs and it was followed by TUKEY assay. An $\alpha=0.05$ was chosen to define significant differences. All numerical data were expressed as mean \pm SEM and all data in graphs were expressed as mean.

\section{Results}

\subsection{Developmental Effects of MMI Treatment on Thyroid Hormone Levels}

As mentioned previously, MMI induces hypothyroidism by inhibiting thyroid iodination and has been as a typical thyroid antagonist. Exposure of pregnant rat to 50ppm, $75 \mathrm{ppm}$ an $100 \mathrm{ppm}$ from GD 3 to PN20 significantly reduced in dose-dependent manner circulating total T3, total T4, free T4 and TSH levels in the dams at developmental stage, but these reductions reversed in hypothyroid with T4-replacement group. All alteration were significantly lower in treatment group (all $\mathrm{p}<0.05$ ). The data of the circulating total $\mathrm{T} 3$ and total and free $\mathrm{T} 4$ and $\mathrm{TSH}$ levels were presented as mean $\pm \mathrm{SD}$ in table 2 and expressed as mean in Figure 1.

Table 2. Plasma tT4, fT4, T3 and TSH of dams. All data were expressed as mean \pm SD.

\begin{tabular}{|c|c|c|c|}
\hline Treatment Group & $\mathrm{T} 3$ (ng/dl) & freeT4(ng/dl) & totalT4 $(\mathrm{ng} / \mathrm{ml})$ \\
\hline Control(n=12) & $1.158 \pm 0.137$ & $0.883 \pm 0.083$ & $45.425 \pm 3.494$ \\
\hline $50 \operatorname{PPM}(\mathrm{n}=12)$ & $0.9500 \pm 0.206$ & $0.5458 \pm 0.307$ & $22.775 \pm 3.721$ \\
\hline $75 \mathrm{ppm}(\mathrm{n}=12)$ & $0.858 \pm 0.131$ & $0.575 \pm 0.2701$ & $22.775 \pm 3.721$ \\
\hline $100 \operatorname{ppm}(\mathrm{n}=12)$ & $0.683 \pm 0.133$ & $0.375 \pm 0.0965$ & $17.65 \pm 1.405$ \\
\hline $50 \mathrm{PPM}$ MMI $+200 \mu \mathrm{gT} 4(\mathrm{n}=12)$ & $0.7917 \pm 0.156$ & $1.313 \pm 0.238$ & $71.175 \pm 12.81$ \\
\hline
\end{tabular}



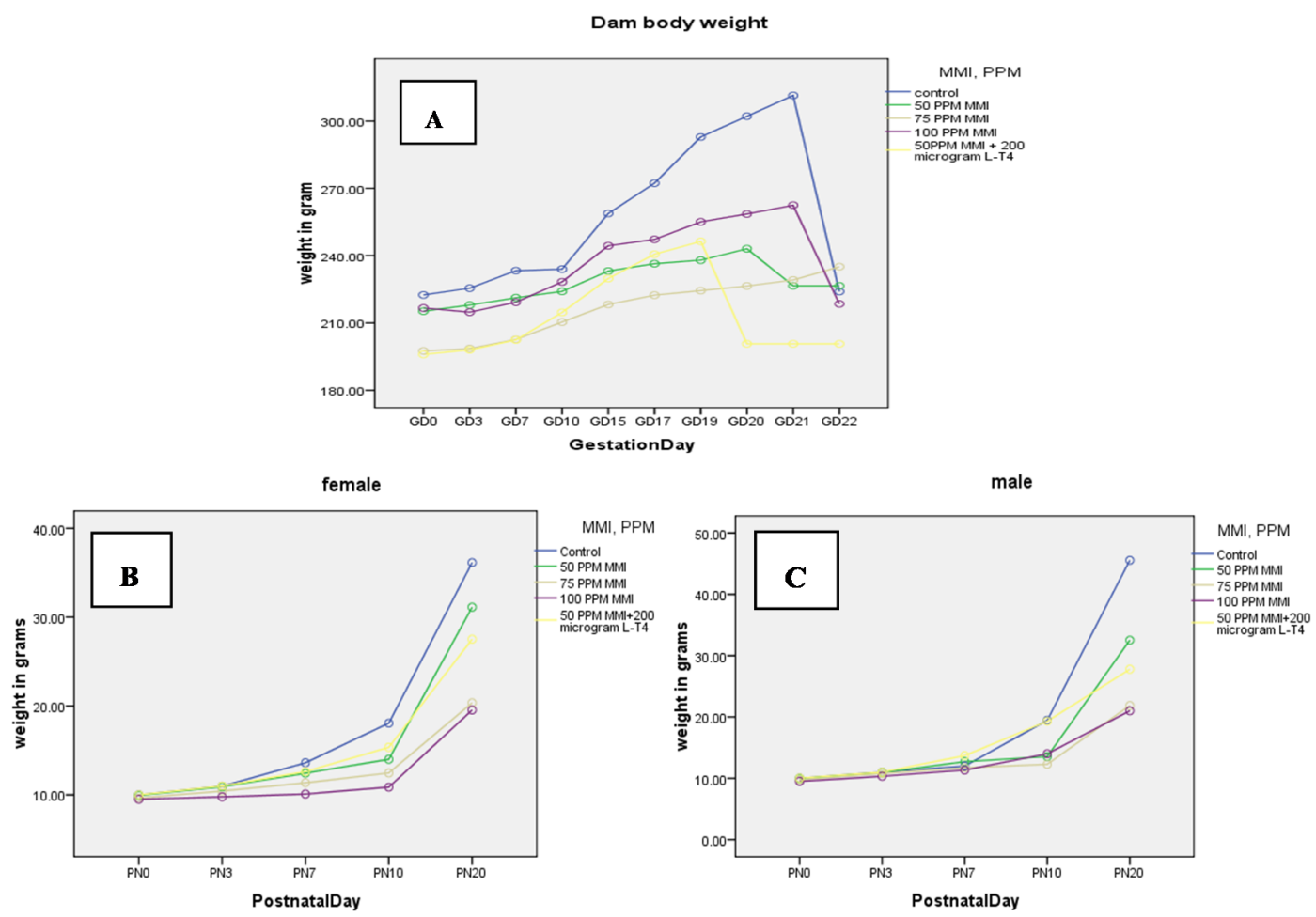

Figure 2. Mean total T3 (ng/dl), total T4 (ng/ml), and free T4 (ng/ dl) in plasma of dams in pup's postnatal day(PN) 20 . Thyroid hormones are dose-dependently reduced by MMI in dams during experimental period in MMI-exposed groups (n=12/group). In MMI-exposed groups total T4, T3 and free T4 significantly different from the control group $(\mathrm{p}<0.05)$. T4 and T3 in hypothyroid with T4-replacement increased ( $\mathrm{n}=12 /$ group). All data in graphs expressed as mean
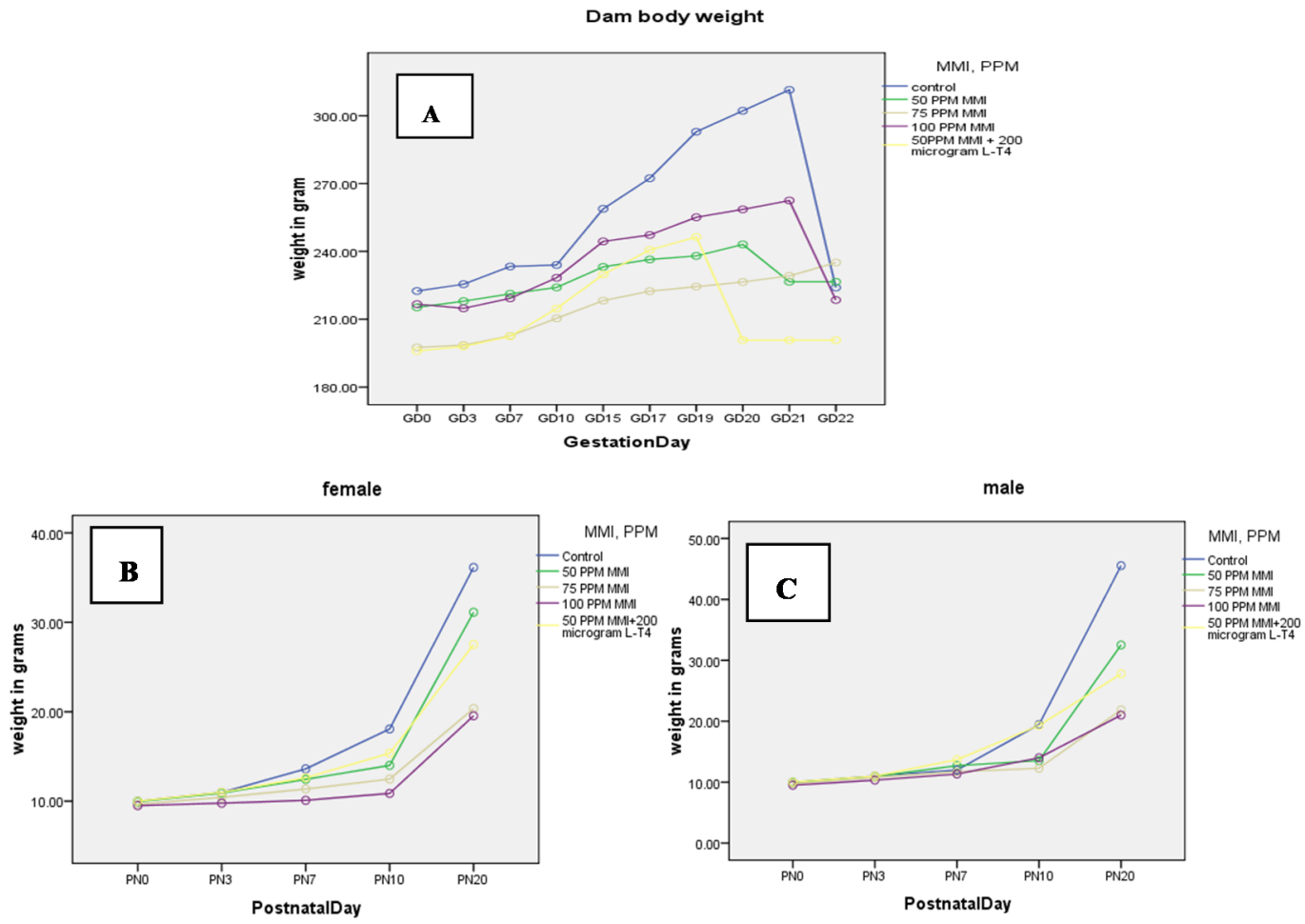

Figure 3. (A) Mean body weight of dams in MMI-exposed dams which were comparable to control ( $n=12$ /group). (B,C) In treated groups Pup's body weight deficits (mean) were evident in pups at sexuality level at PN3 and became profound with age 


\subsection{Body Weight in Dams and Pups}

Among the three experimental groups of control, hypothyroid (MMI-treated), and hypothyroid with T4replacement, there was significant alterations in daily body weight of dams $(F=4.476, p<0.001)$. Body weight deficits (mean) were evident in pups at sexuality level at PN3 and became profound with age ( $F=9.971, p<0.001)$. Pups in the T4-replacement group showed a significant recovery of the growth curve compared with hypothyroid group.

\subsection{Number of Pups at Birth}

The number of pups at birth were evaluated at each breeding. Significant reductions in Number of pups at birth were evident in hypothyroid groups $(\mathrm{p}<0.001)$.

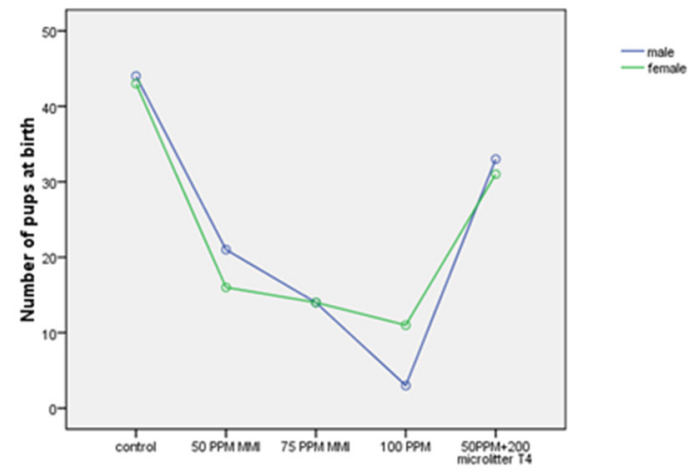

Figure 4. Evaluation of mothers thyroid state on Numbers of pups at birth. In hypothyroid groups the number of pups at each breeding were decreased significantly $(p<0.001)$. In L-T4 treated hypothyroid group the number of pups at birth were recovered

\subsection{Eye-Closure Opening Day}

The neurodevelopment of pups was evaluated indirectly by the day on which the pups opened their eyes. Significant delayed in day the pups opened their eyes were evident in hypothyroid groups $(\mathrm{p}<0.001)$. In L-T4 treated hypothyroid group Day the pups opened their eyes were recovered.

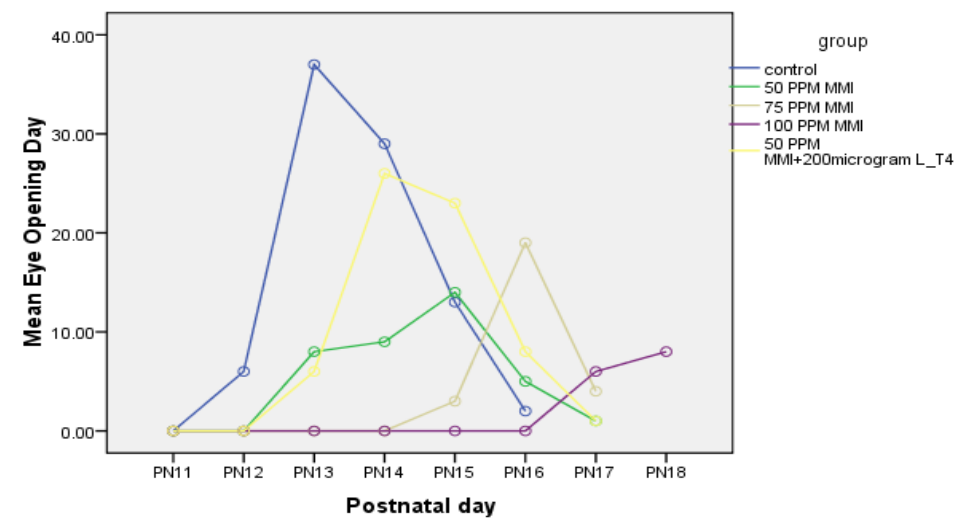

Figure 5. mean Day the pups opened their eyes. In hypothyroid groups Day the pups opened their eyes were delayed significantly $(p<0.001)$. In L-T4 treated hypothyroid group Day the pups opened their eyes. were recovered

\subsection{BDNF Expression varies by Dose of MMI}

Absolute BDNF values were altered in hypothyroid group as a function of exposure to MMI. Statistical analyses revealed significant main effects of MMI in mature BDNF level in whole brain in hypothyroid groups. BDNF levels were significantly decreased in the hypothyroid group $(p<0.001)$. 


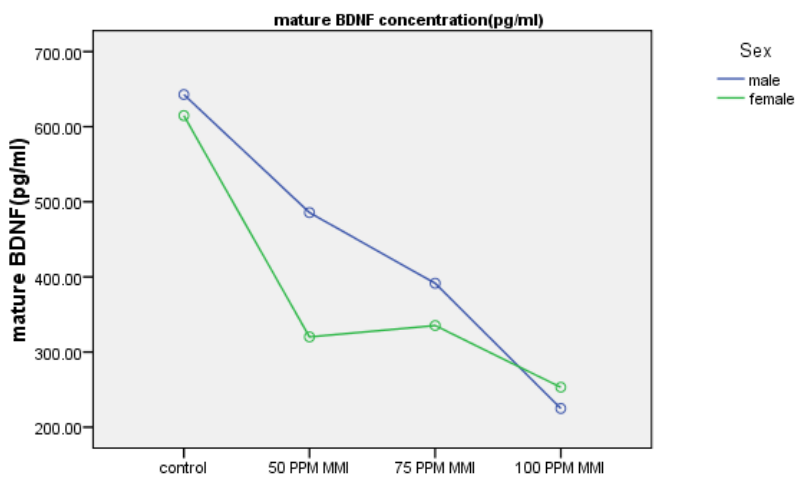

Figure 6. Effect of MMI on mature BDNF levels in the Wistar rat's pups in PN20. BDNF levels were significantly decreased in the hypothyroid group $(p<0.001)$

\subsection{Sensitivity of Gene Expression at Hypothyroid and T4-Replacement Hypothyroid Groups}

The effects of TH deficiency on the relative abundances of nerve growth factor (NGF), brain-derived neurotrophic factor (BDNF), and neurotrophin-3 (NT-3) mRNA were determined by quantitative RT-PCR analysis in cerebellum, medulla, hippocampus, cortex, and olfactory bulbs of pups at PN20. Relative expression of each gene in different brain region of pup's brain at PN20 was compared pairwise between hypothyroid and T4-replacement groups with control.

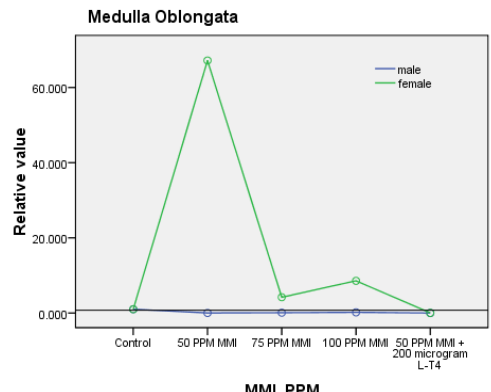

MMI, PPM

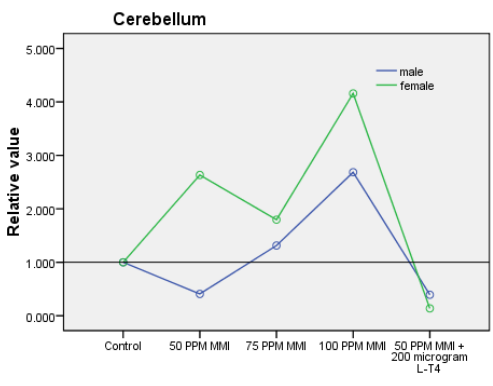

MMI, PPM

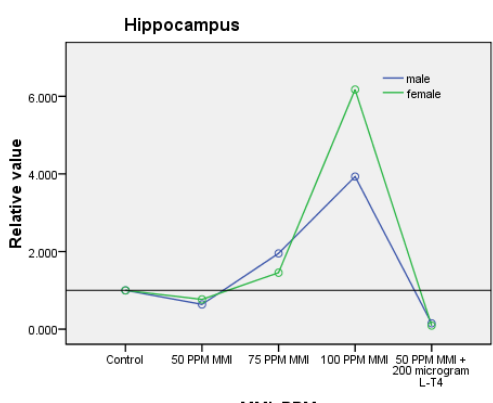

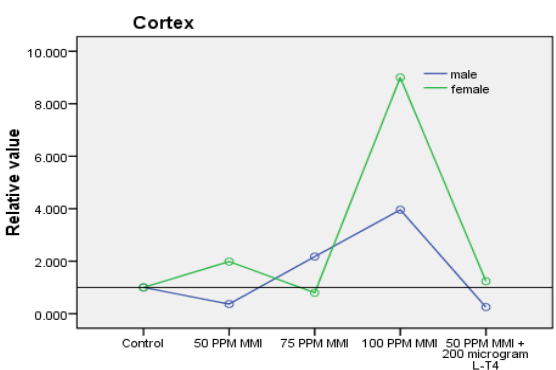

MMI, PPM

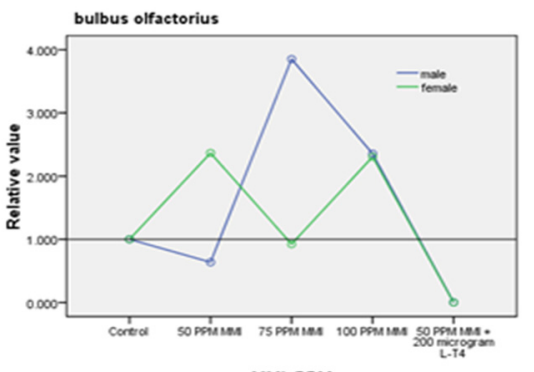

MMI, PPM

Figure 7. Dose-effect relationships for BDNF mRNA relative expression in male and female offspring in each brain region. BDNF mRNA relation values are normalized to the control (0 PPM value) for the respective gender in that brain area. Values are expressed as mean 
Neurotrophins play important roles in neural differentiation, dendritic growth and neuronal migration. Thyroid hormone insufficiency during development impact of BDNF, NGF, and NT3 expression during PN20 in any of the five brain regions assessed. We demonstrated that in male offspring in 50 PPM MMI-treated group and T4replacement hypothyroid group in five brain regions BDNF mRNA relative expression was decreased but in severe hypothyroid groups its relative expression was increased. In female offspring its expression was increased but in T4-replacement hypothyroid group was decreased. BDNF mRNA in cortex and hippocampus of male and female offspring in severe hypothyroid groups increased more than other brain region. Significant sex differences in relative BDNF mRNA was demonstrated in treated groups and in female offspring its relative expression was more than male offspring.

\section{Discussion}

In this study we used an animal model to study the role of thyroid hormones (THs) depletion on developing rat brain.

A number of observations resulted from this investigation were 1) decreased BDNF expression in the neonate of hypothyroid group, 2) but surprisingly, increased BDNF mRNA in the male and female offspring, 3) neurotrophin mRNA expression varies by sex, brain region and dose of MMI, 4) in hypothyroid groups the number of pups at birth was decreased, 5) infant body weight was decreased and 6) the eye closure opening day was delayed.

Dose-dependent thyroid hormone insufficiency in dams was induced by methimazole (MMI). MMI inhibits the enzyme thyroperoxidase, which normally acts in thyroid hormone synthesis by oxidizing the anion iodide $\left(\mathrm{I}^{-}\right)$to iodine (I2), hypoiodous acid (HOI), enzyme linked hypoiodate (EOI) facilitating iodine's addition to tyrosine residues on the hormone precursor thyroglobulin, a necessary step in the synthesis of triiodothyronine (T3) and thyroxine (T4). During pregnancy MMI is widely believed to cross the placenta and accumulate and disrupt fetal thyroid gland (Koren \& Soldin, 2006; Motonaga et al., 2016).

\subsection{Maternal Hypothyroidism on Number of Pups at Birth and Infant Weight}

In maternal thyroid hormone depletion groups the weight of fetus and number of pups at birth was reduced. The alternation observed in groups with thyroid hormone depletion, at least in part, related to the placental development. THs play an essential role in trophoblast function and placental development. Thyroid hormone signaling is involved in the bi-directional dialogue between competent blastocyst and endometrium (Colicchia et al., 2014).Thyroid hormone has been shown to exert direct effects on trophoblast endocrine function (Maruo et al., 1991) and acts a physiological amplifier of trophoblast function and stimulates the functional differentiation of trophoblasts during early pregnancy (Ohara et al., 2004). Thyroid hormone enhances the production of an epidermal growth factor (EGF)-like protein (Matsuo et al., 1993), P4, hCG and hPL in cultured early placental trophoblasts (Maruo et al., 1991) and increased the number of invading cells through Matrigel transwells as well as the expression of cell adhesion molecules, including matrix metalloproteinase-2, -3, fetal fibronectin, and integrin $\alpha 5 \beta 1(\mathrm{Oki}$ et al., 2004).THs exert a protective mechanism at placental level, reducing trophoblastic apoptosis and Fas/Fas-ligand expression(Colicchia et al., 2014). Thyroid hormone has been shown to suppress apoptosis in trophoblast cells by decreasing expression of TNF- $\alpha$ and $F A S$ and Fas ligand genes (Laoag-Fernandez et al., 2004). In the same cells, the hormone decreased the activity of caspase-3, another anti-apoptotic action (Lin et al., 2015). Thyroid hormone depletion leading to the decreased endocrine activity of trophoblast cells and increased their apoptosis. The migration of trophoblast cells is affected by thyroid dysfunction, particularly in hypothyroidism (Laoag-Fernandez et al., 2004). Maternal hypothyroidism reduced the weight of fetuses and altered the thickness of the placental labyrinth and decreased VEGF expression, and increased the apoptotic rate in the placental disk. In other words maternal hypothyroidism affects fetal weight by altering the proliferative activity, apoptosis and vascularization of the placenta (Silva et al., 2012). Fetal growth restriction in hypothyroidism is associated with changes in proliferative activity, apoptosis and vascularization of the placenta (Silva et al., 2012).

\subsection{Maternal Hypothyroidism on the Eye-Closure Opening Day}

We observed that in Tyroid hormone depletion groups eye closure opening days was delayed. Gamborino MJ. et al (2001) showed that in the hypothyroid groups with respect to the controls, on embryological day 13 (E13), values for head parameters, optic primordia area and volume significantly reduced and a delayed prenatal eye closure and postnatal eye opening took place in the treated rats. The photoreceptor and ganglion cell layer thickness displayed significantly lower in HG, at each developmental time point. Postnatally, a delay in photoreceptor outer segment morphogenesis (in relation to retarded disc formation) and significantly lower values for ganglion cell nuclear volumes and nuclear pore density were observed in the TH-deficient animals (Gamborino et al., 2001).Thyroid hormones play an essential role in neuro-retinogenesis (Pinazo-Durán et al., 2011) and maternal 
hypothyroidism during the gestation and suckling period results in eye size, delayed retinal development and morphological abnormalities as well as thinning of the retinal layers (Arbogast et al., 2016). In the THD optic nervous glial development and myelination was significantly delayed as compared to controls (Pinazo-Durán et al., 2011). In wistar rat model Maria D.Pinazo-Durán et al., (2005) showed that TH deprivation altered the organization of the retina (Pinazo-Durán et al., 2005). Low T(3)/T(4) levels delayed and/or altered a series of developmental processes occurring in the retina during the perinatal stage such as layering and differentiation of several cell types(Sevilla-Romero et al., 2002), retinal cell proliferation and cell fate decisions (Harpavat \& Cepko, 2003), craniofacial and ocular proper development (Bohnsack \& Kahana, 2013) and the hypothyroid pups open their eyes later than the euthyroid pups (Pineda-Reynoso et al., 2010). Our results in consistent with this results and showed that in hypothyroid groups eye opening day was delayed and this day was recovered in T4-replacement groups.

\subsection{Maternal and Prenatal Hypothyroidism on BDNF Expression}

Neurtrophic factors including nerve growth factor (NGF), brain-derived neurotrophic factor (BDNF), neurotrophin (NT)-3 and NT-4/5, play critical roles in TH-regulated brain development (Koibuchi \& Iwasaki, 2006). In hypothyroid group thyroid hormone insufficiency resulted in BDNF protein expression. This finding is consistent with some reports in which thyroid hormone depletion in rats led to alternations in BDNF protein. The observations of altered BDNF protein and gene expression have varied. We observed that in five brain regions at hypothyroid group despite significant reductions in thyroid hormones BDNF mRNA not accompanied by changes in BDNF protein and was increased. We analyzed RNA- binding protein HUD mRNA by RT-PCR and observed that HUD mRNA was decreased in all brain region (data not shown). HUD interacts directly with sequences in the long $B d n f$ 3' untranslated region (3'UTR) and co-localizes with $B d n f$ mRNA in dendrites and increased dendritic translation of BDNF (36-37). Sui et al. (2010) induced an animal model of hypothyroidism by administering $0.05 \%(\mathrm{w} / \mathrm{v})$ prophylthiouracil (PTU) in the drinking water to their mothers from 15th day of conception. Some hypothyroid pups received daily injections of T4 $(0.02 \mu \mathrm{g} / \mathrm{g}$ body weight) dissolved in saline from day 1 after birth (postnatal day 1) until death. They observed that hypothyroidism- induced up-regulation in DNA methylation and downregulation in histone $\mathrm{H} 3$ and $\mathrm{H} 4$ acetylation at $B D N F$ promoter and decreased in the $B D N F$ exon II mRNA and protein expression and this led in hypothyroid group $B D N F$ mRNA was lower than euthyroid group. They revealed that suppression of BDNF II exon mRNA and protein expression in rat hippocampus induced by perinatal hypothyroidism was only found at the early developmental stage and epigenetic mechanism of DNA methylation and histone acetylations in BDNF exon II expression at the later developmental stage may not critical. At PN15 BDNF exon II mRNA in hypothyroid and euthyroid group were increased but its relative expression in hypothyroid group was lower than euthyroid group (Sui \& $\mathrm{Li}, 2010$ ). The subclinical model also addressed by Lasley et al., (2011) by administering $0,1,2$ or $3 \mathrm{ppm}$ of thyroid hormone synthesis inhibitor PTU to the drinking water at beginning on GD6 and continuing until postnatal day (PN) 21. They show that in the high dose cohort, despite the $10 \mathrm{ppm}$ dose of PTU inducing hypothyroidism, BDNF protein expression was not reduced in male or female offspring on PN21, but altered BDNF expression profiles present in the hippocampus and cortex of adults offspring. This finding in the $B D N F$ mRNA and protein expression stand in contrast to our results. Sex differences in basal levels of BDNF protein first reported by Lasley et al. (2011). They observed higher levels in adult males than in adult females in hippocampus and cortex with no gender difference evident in cerebellum (Lasley \& Gilbert, 2011). In this study we observed sex differences in total mature BDNF protein and identified that BDNF expression profiles in five brain region varies by sex. At medulla, cerebellum, hippocampus and cortex BDNF expression profiles in female offspring was higher than male offspring but in bulbus olfactorius its expression was reversed. This sex-dependential sensitivity may be due to estrogenic regulation of BDNF expression.

In summary, perinatal thyroid hormone insufficiency on $B D N F$ gene transcription and BDNF protein expression was observed. Any disruption in brain critical proteins and their related signaling pathways result severe neurological, Neurofacial and retinal deficits.

\section{References}

Arbogast, P., Flamant, F., Godement, P., Glösmann, M., \& Peichl, L. (2016). Thyroid hormone signaling in the mouse retina. PloS one, 11(12), e 0168003.

Bernal, J. (2007). Thyroid hormone receptors in brain development and function. Nature clinical practice Endocrinology \& metabolism, 3(3), 249-259.

Bohnsack, B. L., \& Kahana, A. (2013). Thyroid hormone and retinoic acid interact to regulate zebrafish craniofacial neural crest development. Developmental biology, 373(2), 300-309. 
Chen, L. M., Du, W. J., Dai, J., Zhang, Q., Si, G. X., Yang, H., ... Lu, X. M. (2014). Effects of subclinical hypothyroidism on maternal and perinatal outcomes during pregnancy: a single-center cohort study of a Chinese population. PloS one, 9(10), e109364.

Colicchia, M., Campagnolo, L., Baldini, E., Ulisse, S., Valensise, H., \& Moretti, C. (2014). Molecular basis of thyrotropin and thyroid hormone action during implantation and early development. Human reproduction update, 20(6), 884-904.

Friesema, E. C., Ganguly, S., Abdalla, A., Fox, J. E. M., Halestrap, A. P., \& Visser, T. J. (2003). Identification of monocarboxylate transporter 8 as a specific thyroid hormone transporter. Journal of Biological Chemistry, 278(41), 40128-40135.

Friesema, E. C., Jansen, J., Jachtenberg, J. W., Visser, W. E., Kester, M. H., \& Visser, T. J. (2008). Effective cellular uptake and efflux of thyroid hormone by human monocarboxylate transporter 10. Molecular endocrinology, 22(6), 1357-1369.

Gamborino, M. J., Sevilla-Romero, E., Muñoz, A., Hernández-Yago, J., Renau-Piqueras, J., \& Pinazo-Durán, M. D. (2001). Role of thyroid hormone in craniofacial and eye development using a rat model. Ophthalmic research, 33(5), 283-291.

Ghassabian, A., Bongers-Schokking, J. J., Henrichs, J., Jaddoe, V. W., Visser, T. J., Visser, W., ... \& Verhulst, F. C. (2011). Maternal thyroid function during pregnancy and behavioral problems in the offspring: the generation R study. Pediatric research, 69, 454-459.

Gilbert, M. E., \& Lasley, S. M. (2013). Developmental thyroid hormone insufficiency and brain development: a role for brain-derived neurotrophic factor (BDNF)?. Neuroscience, 239, 253-270.

Gilbert, M. E., \& Zoeller, R. T. (2010). Thyroid hormone - impact on the developing brain: possible mechanisms of neurotoxicity. Neurotoxicology: Target organ toxicology series, 79-111.

Gilbert, M., Oshiro, W., Spring, S., Hotchkiss, M., Korte, J., Kosian, P., \& Degitz, S. (2015). Structural abnormalities and learning impairments induced by low level thyroid hormone insufficiency: A crossfostering study. Neurotoxicology and Teratology, (49), 131-132.

Harpavat, S., \& Cepko, C. L. (2003). Thyroid hormone and retinal development: an emerging field. Thyroid, 13(11), 1013-1019.

Huang, E. J., \& Reichardt, L. F. (2001). Neurotrophins: roles in neuronal development and function. Annual review of neuroscience, 24(1), 677-736.

Koibuchi, N., \& Iwasaki, T. (2006). Regulation of brain development by thyroid hormone and its modulation by environmental chemicals. Endocrine journal, 53(3), 295-303.

Koren, G., \& Soldin, O. (2006). Therapeutic drug monitoring of antithyroid drugs in pregnancy: the knowledge gaps. Therapeutic drug monitoring, 28(1), 12.

Laoag-Fernandez, J. B., Matsuo, H., Murakoshi, H., Hamada, A. L., Tsang, B. K., \& Maruo, T. (2004). 3, 5, 3'Triiodothyronine down-regulates Fas and Fas ligand expression and suppresses caspase-3 and poly (adenosine 5'-diphosphate-ribose) polymerase cleavage and apoptosis in early placental extravillous trophoblasts in vitro. The Journal of Clinical Endocrinology \& Metabolism, 89(8), 4069-4077.

Lasley, S. M., \& Gilbert, M. E. (2011). Developmental thyroid hormone insufficiency reduces expression of brainderived neurotrophic factor (BDNF) in adults but not in neonates. Neurotoxicology and teratology, 33(4), 464-472.

Lin, H. Y., Glinsky, G. V., Mousa, S. A., \& Davis, P. J. (2015). Thyroid hormone and anti-apoptosis in tumor cells. Oncotarget, 6(17), 14735.

Maruo, T., Matsuo, H., \& Mochizuki, M. (1991). Thyroid hormone as a biological amplifier of differentiated trophoblast function in early pregnancy. Acta Endocrinologica, 125(1), 58-66.

Matsuo, H., Maruo, T., Murata, K., \& Mochizuki, M. (1993). Human early placental trophoblasts produce an epidermal growth factor-like substance in synergy with thyroid hormone. Acta endocrinologica, 128(3), 225229.

Morreale de Escobar, G., Jesús Obregón, M., \& Escobar del Rey, F. (2000). Is neuropsychological development related to maternal hypothyroidism or to maternal hypothyroxinemia?. The Journal of Clinical Endocrinology \& Metabolism, 85(11), 3975-3987. 
Motonaga, K., Ota, M., Odawara, K., Saito, S., \& Welsch, F. (2016). A comparison of potency differences among thyroid peroxidase (TPO) inhibitors to induce developmental toxicity and other thyroid gland-linked toxicities in humans and rats. Regulatory Toxicology and Pharmacology, 80, 283-290.

Ohara, N., Tsujino, T., \& Maruo, T. (2004). The role of thyroid hormone in trophoblast function, early pregnancy maintenance, and fetal neurodevelopment. Journal of Obstetrics and Gynaecology Canada, 26(11), 982-990.

Oki, N., Matsuo, H., Nakago, S., Murakoshi, H., Laoag-Fernandez, J. B., \& Maruo, T. (2004). Effects of 3, 5, 3'triiodothyronine on the invasive potential and the expression of integrins and matrix metalloproteinases in cultured early placental extravillous trophoblasts. The Journal of Clinical Endocrinology \& Metabolism, 89(10), 5213-5221.

Pinazo-Durán, M. D., Iborra, F. J., Pons, S., Sevilla-Romero, E., Gallego-Pinazo, R., \& Muñoz, A. (2005). Postnatal thyroid hormone supplementation rescues developmental abnormalities induced by congenitalneonatal hypothyroidism in the rat retina. Ophthalmic research, 37(4), 225-234.

Pinazo-Durán, M. D., Pons-Vázquez, S., Gallego-Pinazo, R., Estrada, C. G., Zanón-Moreno, V., Bou, V. V., \& Solana, P. S. (2011). Thyroid hormone deficiency disrupts rat eye neurodevelopment. Brain research, 1392, 16-26.

Pineda-Reynoso, M., Cano-Europa, E., Blas-Valdivia, V., Hernandez-Garcia, A., Franco-Colin, M., \& OrtizButron, R. (2010). Hypothyroidism during neonatal and perinatal period induced by thyroidectomy of the mother causes depressive-like behavior in prepubertal rats. Neuropsychiatric disease and treatment, 6, 137.

Royland, J. E., Parker, J. S., \& Gilbert, M. E. (2008). A genomic analysis of subclinical hypothyroidism in hippocampus and neocortex of the developing rat brain. Journal of neuroendocrinology, 20(12), 1319-1338.

Sevilla-Romero, E., Muñoz, A., \& Pinazo-Durán, M. D. (2002). Low thyroid hormone levels impair the perinatal development of the rat retina. Ophthalmic research, 34(4), 181-191.

Silva, J. F., Vidigal, P. N., Galvão, D. D., Boeloni, J. N., Nunes, P. P., Ocarino, N. M., ... \& Serakides, R. (2012). Fetal growth restriction in hypothyroidism is associated with changes in proliferative activity, apoptosis and vascularisation of the placenta. Reproduction, Fertility and Development, 24(7), 923-931.

Stenzel, D., Wilsch-Bräuninger, M., Wong, F. K., Heuer, H., \& Huttner, W. B. (2014). Integrin $\alpha v \beta 3$ and thyroid hormones promote expansion of progenitors in embryonic neocortex. Development, 141(4), 795-806.

Sui, L., \& Li, B. M. (2010). Effects of perinatal hypothyroidism on regulation of reelin and brain-derived neurotrophic factor gene expression in rat hippocampus: role of DNA methylation and histone acetylation. Steroids, 75(12), 988-997.

Warner, A., \& Mittag, J. (2012). Thyroid hormone and the central control of homeostasis. Journal of molecular endocrinology, 49(1), R29-R35.

Zoeller, R. T., \& Rovet, J. (2004). Timing of thyroid hormone action in the developing brain: clinical observations and experimental findings. Journal of neuroendocrinology, 16(10), 809-818.

\section{Copyrights}

Copyright for this article is retained by the author(s), with first publication rights granted to the journal.

This is an open-access article distributed under the terms and conditions of the Creative Commons Attribution license (http://creativecommons.org/licenses/by/4.0/). 